\title{
Response by the Authors of Original Article
}

\author{
Beth N. McNulty ${ }^{1}$ Sean Wise ${ }^{2}$ David S. Cohen ${ }^{3}$ \\ ${ }^{1}$ Department of Otology and Neurotology, Michigan Ear Institute, \\ Farmington Hills, Michigan, United States \\ 2 Department of Otology, Neurotology and Skull Base Surgery, \\ Naval Medical Center, San Diego, California, United States \\ 3 Department of Otolaryngology, Wayne State University, Detroit, \\ Michigan, United States \\ ${ }^{4}$ Department of Otolaryngology, Loyola University, Maywood, \\ Illinois, United States \\ ${ }^{5}$ Department of Otology, Neurotology and Skull Base Surgery, \\ Michigan Ear Institute, Farmington Hills, Michigan, United States
}

J Neurol Surg B 2019;80:329.

We would like to thank the authors of the letter to the editor regarding this study for calling attention to an additional imaging modality that may aid in distinguishing between tumors of facial nerve origin and vestibular schwannomas. The objective of our study was to identify both preoperative and intraoperative tools to better distinguish facial schwannomas from vestibular schwannomas. The authors suggested that diffusion tensor tractography may be used to establish a relationship between facial nerve fibers and tumor. It was asked that we clarify the imaging protocol for our institution. The patients, in this study, were evaluated using our institutional imaging protocol of preoperative magnetic resonance imaging with and without gadolinium contrast. We do not employ intraoperative neuronavigation in these cases. The authors quote two studies that have shown diffusion tensor tractography to be valuable in predicting the location of the facial nerve in relation to the tumor. ${ }^{1,2}$ While this imaging tool is gaining popularity among skull base surgeons, a recent systematic review found only 14 studies documenting its use in vestibular schwannoma resection. The review concluded that it is indeed a useful adjunct in large vestibular schwannomas, size $>2.5 \mathrm{~cm}$, successfully identifying the facial nerve course in $96.6 \%$ of tumors with an accuracy of
Address for correspondence Beth N. McNulty, MD, Department of Otolaryngology Head and Neck Surgery, University of Kentucky College of Medicine, Kentucky Clinic Ste. B-317, 740 South Limestone Street, Lexington, KY 40536, United States (e-mail: bnmcnulty@gmail.com).

$90.6 \%^{3}$. We agree that this may be a useful tool in the future but recognize that ongoing research is needed to evaluate the costeffectiveness and to further establish a direct benefit to facial nerve outcome with its use.

\section{Conflicts of Interest}

There are no financial disclosures or conflicts of interest to disclose.

\section{References}

1 Chen DQ Quan J, Guha A, Tymianski M, Mikulis D, Hodaie M. Three-dimensional in vivo modeling of vestibular schwannomas and surrounding cranial nerves with diffusion imaging tractography. Neurosurgery 2011;68(04):1077-1083

2 Yoshino M, Kin T, Ito A, et al. Feasibility of diffusion tensor tractography for preoperative prediction of the location of the facial and vestibulocochlear nerves in relation to vestibular schwannoma. ActaNeurochir (Wien) 2015;157(06):939-946, discussion 946

3 Savardekar AR, Patra DP, Thakur JD, et al. Preoperative diffusion tensor imaging-fiber tracking for facial nerve identification in vestibular schwannoma: a systematic review on its evolution and current status with a pooled data analysis of surgical concordance rates. Neurosurg Focus 2018;44(03):E5 published online January 24, 2019 (c) 2019 Georg Thieme Verlag KG

Stuttgart · New York
DOI https://doi.org/ 10.1055/s-0038-1676833. ISSN 2193-6331. 